\title{
Alternating Sign Matrices and Polynomiography
}

\author{
Bahman Kalantari \\ Department of Computer Science \\ Rutgers University, USA \\ kalantari@cs.rutgers.edu
}

Submitted: Apr 10, 2011; Accepted: Oct 15, 2011; Published: Oct 31, 2011

Mathematics Subject Classifications: 00A66, 15B35, 15B51, 30C15

Dedicated to Doron Zeilberger on the occasion of his sixtieth birthday

\begin{abstract}
To each permutation matrix we associate a complex permutation polynomial with roots at lattice points corresponding to the position of the ones. More generally, to an alternating sign matrix (ASM) we associate a complex alternating sign polynomial. On the one hand visualization of these polynomials through polynomiography, in a combinatorial fashion, provides for a rich source of algorithmic art-making, interdisciplinary teaching, and even leads to games. On the other hand, this combines a variety of concepts such as symmetry, counting and combinatorics, iteration functions and dynamical systems, giving rise to a source of research topics. More generally, we assign classes of polynomials to matrices in the Birkhoff and ASM polytopes. From the characterization of vertices of these polytopes, and by proving a symmetry-preserving property, we argue that polynomiography of ASMs form building blocks for approximate polynomiography for polynomials corresponding to any given member of these polytopes. To this end we offer an algorithm to express any member of the ASM polytope as a convex of combination of ASMs. In particular, we can give exact or approximate polynomiography for any Latin Square or Sudoku solution. We exhibit some images.
\end{abstract}

Keywords: Alternating Sign Matrices, Polynomial Roots, Newton's Method, Voronoi Diagram, Doubly Stochastic Matrices, Latin Squares, Linear Programming, Polynomiography

\section{Introduction}

Polynomials are undoubtedly one of the most significant objects in all of mathematics and the sciences, particularly in combinatorics. There are famous polynomials or classes of polynomials in many fields of mathematics. While working with a real or complex 
polynomial does not necessarily require dealing with its roots, the roots define the polynomial and are thus implicitly present in any application. For many years this author's research has been devoted to the study of polynomial root-finding and their algorithmic visualization via iteration functions for which a term has been coined, polynomiography. For instance, the behavior of Newton's method in the complex plane as applied to the cubic roots of unity, initially investigated by Cayley [4] in 1879, is well-known to give rise to a fractal image, which in our terminology is a polynomiograph, or more precisely a fractal polynomiograph. Newton's iteration function is only one member of infinite classes of iteration functions and some particular applications of these lead to polynomiographs that are not fractal in nature. This and other reasons justify the definition and legitimacy of polynomiography as a new foundation for polynomial root-finding visualization; see [8] for detailed explanation. In subsequent sections we will define a particular class of iteration functions with significant properties.

This article focuses on polynomiography as applied to a polynomial that we associate to a permutation matrix, an alternating sign matrix (ASM), and more generally to any matrix in the convex hull of permutation matrices, or in the convex hull of the alternating sign matrices. These convex hulls are known as the Birkhoff polytope and the ASM polytope, respectively. The latter has recently been characterized by Striker [20]. Our inspiration behind defining these polynomials lies in the many beautiful results on ASMs presented at Doron Zeilberger's 60th birthday celebration in 2010, in honor of his many accomplishments, including his proof [21] of the famous conjecture of Mills, Robbins, and Rumsey [14], that the number of $n \times n$ alternating sign matrices is

$$
\prod_{j=0}^{n-1} \frac{(3 j+1) !}{(n+j) !} .
$$

The inspiration for the article was sparked by the idea that perhaps by association of certain complex polynomials to permutation matrices, and more generally to ASMs, even for small size matrices, we will have at our disposal a very large class of complex polynomials that could lend themselves to aesthetically pleasing polynomiographs and much else. A preliminary version of this idea, but only for permutation matrices was presented in [9]. While many famous classes of polynomials have real coefficients, association of polynomials to ASMs allows defining complex polynomials that are interesting from the point of view of polynomiography and possibly other perspectives.

Specifically, in Section 2 we first introduce the Basic Family of iteration functions, summarize their basic convergence properties, and briefly describe polynomiography. In Section 3, we consider permutation matrices and to each such matrix of order $n$ we associate a complex polynomial of degree $n$ with roots at lattice points corresponding to the positions of the ones in the matrix. We refer to these as complex permutation polynomials. In Section 4, we consider this in more generality and associate to alternating sign matrices the class of complex alternating sign polynomials. More generally, we assign classes of polynomials to the Birkhoff polytope, and the ASM polytope. In Section 5, we consider doubly stochastic matrices and associate doubly stochastic polynomials. In Section 6 , we prove a symmetry-preserving property of the Basic Family that will be used in two 
different senses: first, it is used to justify the definition of doubly stochastic polynomials and their polynomiography; second, it is used to develop approximate polynomiography for doubly stochastic matrices and ASMs. In Section 7, we consider polynomiography for Latin Squares and Sudoku matrix solutions, where even for small values of $n$ this gives rise to the application of our result to a very large number of vertices. In Section 8, we consider an approach for assigning approximate polynomiography to doubly stochastic matrices based on the decomposition of the corresponding doubly stochastic matrix as the convex combination of permutation matrices. We also describe an algorithm for deriving such decomposition. In Section 9, we associate polynomiography to matrices in the ASM polytope. Furthermore, we extend the decomposition algorithm for doubly stochastic matrices to ASMs. In this section, we also define a concept of diagonally scaling for alternating sign matrices, allowing the assignment of polynomials and polynomiography to even more general matrices than those of ASM polytope. We consider some examples and give the corresponding polynomiography.

On the one hand, visualization of these polynomials through polynomiography, in a combinatorial fashion, provides for a rich source of algorithmic art-making, interdisciplinary teaching, and even leads to games. On the other hand, this combines a variety of concepts such as symmetry, counting and combinatorics, iteration functions and dynamical systems, giving rise to a source of research topics.

\section{The Basic Family and Polynomiography}

In this section we describe the Basic Family of iteration functions for polynomial rootfinding and summarize their relevant properties. Let $p(z)$ be a given complex polynomial of degree $n$. Set $D_{0}(z)=1$, and for each $m \geq 2$ define the $m \times m$ matrix determinant

$$
D_{m}(z)=\operatorname{det}\left(\begin{array}{ccccc}
p^{\prime}(z) & \frac{p^{\prime \prime}(z)}{2 !} & \ldots & \frac{p^{(m-1)}(z)}{(m-1) !} & \frac{p^{(m)}(z)}{(m) !} \\
p(z) & p^{\prime}(z) & \ddots & \ddots & \frac{p^{(m-1)}(z)}{(m-1) !} \\
0 & p(z) & \ddots & \ddots & \vdots \\
\vdots & \vdots & \ddots & \ddots & \frac{p^{\prime \prime}(z)}{2 !} \\
0 & 0 & \ldots & p(z) & p^{\prime}(z)
\end{array}\right) .
$$

The Basic Family then is the collection of iteration functions

$$
B_{m}(z)=z-p(z) \frac{D_{m-2}(z)}{D_{m-1}(z)}, \quad m=2,3, \ldots
$$

Specific members, $B_{2}(z)$ and $B_{3}(z)$, are Newton and Halley iteration functions, respectively. These two by themselves posses a rich and interesting history, see [8]. The Basic Family was studied by Schröder [18]; see also [8] for several different but equivalent formulations, as well as many other properties. The determinant $D_{m}(z)$ can be shown to also satisfy the recurrence

$$
D_{m}(z)=\sum_{i=1}^{n}(-1)^{i-1} p(z)^{i-1} \frac{p^{(i)}(z)}{i !} D_{m-i}(z) .
$$


The following theorem summarizes some relevant properties of the Basic Family. The theorem can be viewed as a particular case of a determinantal Taylor's theorem.

Theorem 1 ([5], [8]) Let $p(z)$ be a complex polynomial of degree $n$, and $\theta$ a root. For each $m \geq 2$, the following expansion is valid

$$
B_{m}(z)=z-p(z) \frac{D_{m-2}(z)}{D_{m-1}(z)}=\theta+(-1)^{m} \sum_{k=m}^{m+n-2} \frac{\widehat{D}_{m-1, k}(z)}{D_{m-1}(z)}(\theta-z)^{k},
$$

where for each $m \geq 1$ and each $k=m+1, \ldots, m+n-1$,

$$
\widehat{D}_{m, k}(z)=\operatorname{det}\left(\begin{array}{ccccc}
\frac{p^{\prime \prime}(z)}{2 !} & \frac{p^{\prime \prime \prime}(z)}{3 !} & \ldots & \frac{p^{(m)}(z)}{(m) !} & \frac{p^{(k)}(z)}{k !} \\
p^{\prime}(z) & \frac{p^{\prime \prime}(z)}{2 !} & \ddots & \frac{p^{(m-1)}(z)}{(m-1) !} & \frac{p^{(k-1)}(z)}{(k-1) !} \\
p(z) & p^{\prime}(z) & \ddots & \vdots & \vdots \\
\vdots & \vdots & \ddots & \frac{p^{\prime \prime}(z)}{2 !} & \frac{p^{(k-m+2)}(z)}{(k-m+2) !} \\
0 & 0 & \cdots & p^{\prime}(z) & \frac{p^{(k-m+1)}(z)}{(k-m+1) !}
\end{array}\right) .
$$

In particular, the expansion formula (4) implies there exists a disk centered at $\theta$ such that for any $z_{0}$ in this disk the sequence of fixed point iteration

$$
z_{k+1}=B_{m}\left(z_{k}\right), \quad k=0,1, \ldots
$$

is well-defined, and it converges to $\theta$. If $\theta$ is a simple root, the order of convergence is $m$. More specifically,

$$
\lim _{k \rightarrow \infty} \frac{\left(\theta-z_{k+1}\right)}{\left(\theta-z_{k}\right)^{m}}=(-1)^{m} \frac{\widehat{D}_{m-1, m}(\theta)}{p^{\prime}(\theta)^{m-1}} .
$$

Definition 1 For a given complex number $w$, the Basic Sequence is defined as

$$
\left\{B_{m}(w), \quad m=2,3, \cdots\right\} .
$$

The Voronoi cell of $\theta, V(\theta)$, is the set of all points in the Euclidean plane that are closer to $\theta$ than any other root of $p(z)$. The following theorem describes one of the powerful properties of the Basic Family.

Theorem 2 ([6], [8]) For any root $\theta$ of $p(z)$ and any $w$ in the Voronoi cell $V(\theta)$, the Basic Family is pointwise convergent to $\theta$, i.e. the corresponding Basic Sequence satisfies:

$$
\lim _{m \rightarrow \infty} B_{m}(w)=\theta
$$

Theorem 2 establishes the pointwise convergence of the Basic Family on each Voronoi cell. However, recently uniform convergence has been proven, thus describing a more accurate and powerful description for this convergence. For this and other recent results on the Basic Family; see [10]. 
To each given polynomial $p(z)$ we associate polynomiography. Polynomiography is the systematic study of the visualization of the process of solving a polynomial equation using iteration functions; see [8]. An individual image under this visualization is called a polynomiograph. It is a two-dimensional computer-generated image that may or may not exhibit fractal behavior. We can think of a polynomiograph as a matrix of computer pixels having a color decided upon by a particular polynomial root-finding algorithm, e.g. Newton's iteration function, or even the collection of the Basic Family as applied to a single polynomial. A color can be identified by a number and it is based on the convergence properties of orbits, basins of attraction, basic sequences, and other criteria. These terms are defined formally below. In a simplest approach, the color of a pixel is dependent upon the root whose basin of attraction contains the location of the pixel.

The individual use of a Basic Family member $B_{m}(z)$ in solving the polynomial equation $p(z)=0$ consists of the fixed point iteration $z_{k+1}=B_{m}\left(z_{k}\right)$, where $z_{0}$ is a starting complex number. The sequence $\left\{z_{k}\right\}_{k=0}^{\infty}$ is called the orbit of $z_{0}$. The basin of attraction of a root $\theta$ is the set of all points whose orbit under the iterations of $B_{m}(z)$ converges to $\theta$. It is an open set and a part of the so-called Fatou set of $B_{m}(z)$. The basin of attraction of each root consists of the union of connected components, called Fatou components. The immediate basin of attraction of a root is the connected component that contains the root. From the local convergence behavior of $B_{m}(z)$ the immediate basin of attraction of a root is well-defined. The boundary of the Fatou components forms the Julia set, known to exhibit fractal behavior for general polynomials. For a precise definition of Fatou and Julia sets with respect to general rational functions and their properties, see e.g. [1], [15], and $[8]$.

Polynomiographs could even inspire 3D images or structures. For a description of polynomiography, its theoretical foundation, artistic applications, as well as various practical and educational applications, see [7], and especially [8]. Polynomiography as theory and software is a novel mathematically inspired computer visualization medium with potentially many applications in education, math, sciences, art and design. In a polynomiograph, whether or not it would exhibit fractal patterns, there could lie either hidden symmetries intrinsic in the choice of its corresponding polynomial, or symmetries that can be created by design. Indeed polynomiography is a rich source for symmetry because in a sense any symmetry in the shape of the roots of a given polynomial, e.g. line symmetry, radial symmetry, rotational symmetry, or homothetic symmetry can be converted into a corresponding form of symmetry in the polynomiographs. What this suggests is that we can create many symmetries simply by the manipulation of a set of points. In this sense, we can create symmetry in a minimalistic fashion. We can reach the very foundation of symmetry in polynomiographs by examining the symmetries in the roots, as well as the mathematical properties of iteration functions under consideration. In this article, we will formally prove a symmetry-preserving property and make use of it in polynomiographies that we will associate to ASMs. 


\section{Permutation Matrices and Polynomiography}

An $n \times n$ permutation matrix $\Pi=\left(\pi_{i j}\right)$ is a matrix whose rows (and columns) form a permutation of the identity matrix. To each permutation matrix, we associate a complex polynomial defined as follows. First, set $\theta_{i j}$ to be the complex number associated to the $(i, j)$ location, $i, j \in\{1, \ldots, n\}$, i.e.

$$
\theta_{i j}=i+\mathbf{i} j, \quad \mathbf{i}=\sqrt{-1}
$$

For a general $n \times n$ matrix $A=\left(a_{i j}\right)$, set

$$
\bar{A}=\left(\bar{a}_{i j}\right), \quad \bar{a}_{i j}=a_{j,(n+1-i)} .
$$

This matrix is analogous to the transpose, except that the $i$-th row of $A$ corresponds to the $i$-th column of $\bar{A}$ but written from bottom up. Now given the matrix $\Pi=\left(\pi_{i j}\right)$ we use the corresponding matrix $\bar{\Pi}=\left(\bar{\pi}_{i j}\right)$ to associate a complex polynomial $P_{\Pi}(z)$ to $\Pi$ defined as

$$
P_{\Pi}(z)=\prod_{\bar{\pi}_{i j}=1}\left(z-\theta_{i j}\right),
$$

a polynomial of degree $n$ referred as the complex permutation polynomial corresponding to $\Pi$.

In this article, we first wish to create polynomiography with permutations polynomials. As an example, for $n=2$ the permutation matrices and their corresponding polynomials are

$$
\left(\begin{array}{ll}
1 & 0 \\
0 & 1
\end{array}\right), \quad\left(\begin{array}{ll}
0 & 1 \\
1 & 0
\end{array}\right), \quad(z-(1+2 i))(z-(2+1 i)), \quad(z-(1+1 i))(z-(2+2 i))
$$

We may associate many polynomiographs to these permutation polynomials. As an example Figure 1 presents two corresponding polynomiographs.
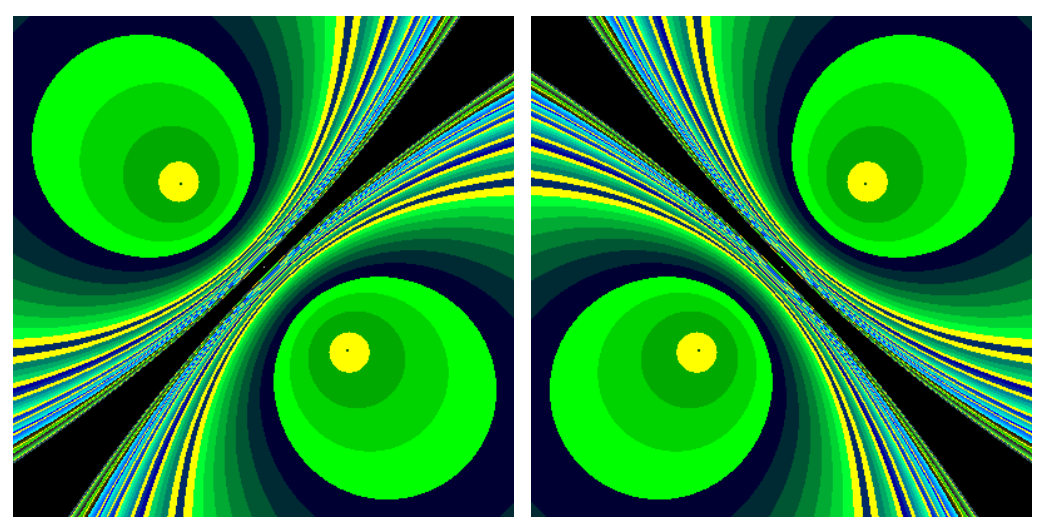

Figure 1: Polynomiographs corresponding to two permutation matrices of order 2. 
For $n=3$ the six permutation matrices and their corresponding polynomials are

$$
\begin{gathered}
\left(\begin{array}{lll}
1 & 0 & 0 \\
0 & 1 & 0 \\
0 & 0 & 1
\end{array}\right),\left(\begin{array}{lll}
1 & 0 & 0 \\
0 & 0 & 1 \\
0 & 1 & 0
\end{array}\right),\left(\begin{array}{lll}
0 & 1 & 0 \\
1 & 0 & 0 \\
0 & 0 & 1
\end{array}\right),\left(\begin{array}{lll}
0 & 0 & 1 \\
1 & 0 & 0 \\
0 & 1 & 0
\end{array}\right),\left(\begin{array}{lll}
0 & 1 & 0 \\
0 & 0 & 1 \\
1 & 0 & 0
\end{array}\right),\left(\begin{array}{ccc}
0 & 0 & 1 \\
0 & 1 & 0 \\
1 & 0 & 0
\end{array}\right) \\
(z-(1+3 i))(z-(2+2 i))(z-(3+i)),(z-(1+3 i))(z-(2+1 i))(z-(3+2 i)), \\
(z-(1+2 i))(z-(2+3 i))(z-(3+i)),(z-(1+2 i))(z-(2+1 i))(z-(3+3 i)), \\
(z-(1+i))(z-(2+3 i))(z-(3+2 i)),(z-(1+i))(z-(2+2 i))(z-(3+3 i)) .
\end{gathered}
$$
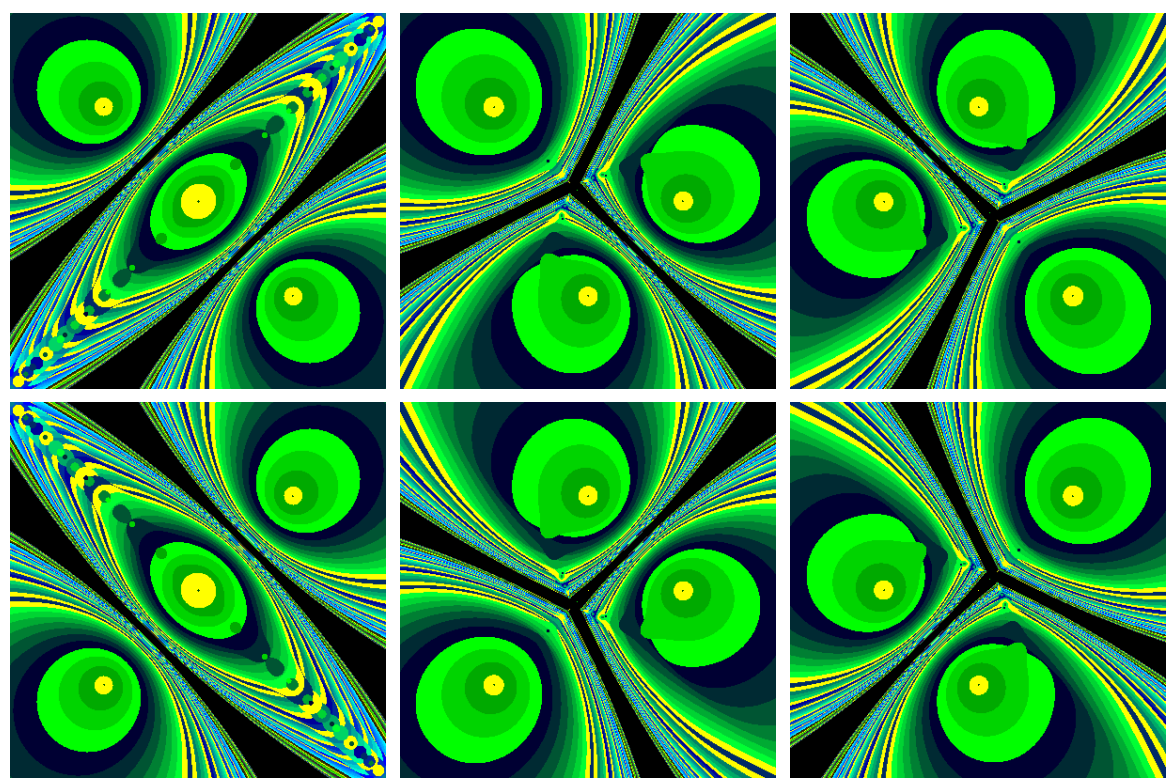

Figure 2: Polynomiographs corresponding to six permutation matrices of order 3.

Figure 2 gives polynomiographs corresponding to 6 permutation matrices of order 3 . These are just a few of the polynomiographies we can associate to these permutation polynomials. Clearly, these polynomiographs exhibit the symmetries appearing in the corresponding permutation matrices.

While the product of two permutation matrices is still a permutation matrix, one may assign polynomiographies to the product of complex permutation polynomials and these could introduce even more interesting images. We will consider a more general approach in Section 5.

\section{Alternating Sign Matrices and Polynomiography}

In this section, we associate a polynomial to each $n \times n$ matrix $A=\left(a_{i j}\right)$ that is an alternating sign matrix (ASM), i.e. its entries are 0 , or \pm 1 ; the sum of entries in each 
row and each column is one; and the nonzero entries in each row and column alternate in sign. As in the case of a permutation matrix, we let

$$
\bar{A}=\left(\bar{a}_{i j}\right), \quad \bar{a}_{i j}=a_{j,(n+1-i)} .
$$

Given, $\theta_{i j}=i+\mathbf{i} j$, we associate a polynomial to $A$ defined as follows

$$
P_{A}(z)=\prod_{a_{i j}=1}\left(z-\theta_{i j}\right) \times \prod_{a_{i j}=-1}\left(z-\theta_{i j}\right)^{2} .
$$

In summary, this gives a generalization of the permutation case and $\theta_{i j}$ is a simple root of $P_{A}$ when $a_{i j}=1$ and a double root when $a_{i j}=-1$. Rather than associating a polynomial to $A$ we could assign the rational function

$$
R_{A}(z)=\prod_{\bar{a}_{i j}=1}\left(z-\theta_{i j}\right) / \prod_{\bar{a}_{i j}=-1}\left(z-\theta_{i j}\right) .
$$

Another alternative would be to associate the polynomial

$$
Q_{A}(z)=\prod_{a_{i j}=1}\left(z-\theta_{i j}\right) \times \prod_{\bar{a}_{i j}=-1}\left(z+\theta_{i j}\right) .
$$

While these alternatives would be interesting and perhaps worth investigating, with regard to polynomiography, working with $R_{A}(z)$ drastically reduces the practicality in applying the Basic Family in an interesting fashion. For instance, we could apply Newton's method efficiently but its polynomiograph would be unremarkable. In the case of $Q_{A}(z)$, the disadvantage lies in that the roots fall outside of an $n$ by $n$ square. The use of double roots allows us to keep the polynomial degree small, yet distinguish between simple and double roots. We refer to the class of polynomials so defined as complex alternating sign polynomials. Figure 3 gives a set of polynomiographs corresponding to the following ASM, taken from the cover of Bressoud's book [3].

$$
\left(\begin{array}{ccccc}
0 & 0 & 1 & 0 & 0 \\
0 & 1 & -1 & 0 & 1 \\
1 & -1 & 0 & 1 & 0 \\
0 & 1 & 0 & 0 & 0 \\
0 & 0 & 1 & 0 & 0
\end{array}\right)
$$

The corresponding polynomial is the product of the terms written columnwise $(z-(1+3 i)),(z-(2+2 i)) *(z-(2+3 i))^{2} *(z-(2+4 i)),(z-(3+i)) *(z-(3+4 i))^{2} *(z-(3+5 i))$, $(z-(z-(4+3 i))$ and $(z-(5+4 i))$. This simplifies into

$$
z^{11}-(30+36 i) z^{10}-(180-979 i) z^{9}+(11029-6201 i) z^{8}-(99937+38130 i) z^{7}+
$$
$(204672+597662 i) z^{6}+(1381808+2274455 i) z^{5}-(7912877-1192177 i) z^{4}+(12604344+$ $11172954 i) z^{3}+(684870-23644850 i) z^{2}-(15679700-12355900 i) z+(7510000+1580000 i)$. 
Rather than working with this polynomial, a symmetry-preserving property in polynomiography (Section 6) allows us to shift the roots while retaining the same polynomiograph. If we shift the origin to the location $(3,3)$ this results in the following polynomial with smaller coefficients

$(z-2 i) *(z+1-i) *(z-i)^{2} *(z-2-i) *(z+2) *(z+1)^{2} *(z-1) *(z+1+i) *(z+2 i)=$ $z^{11}+(3-3 i) z^{10}-11 i z^{9}-(14+18 i) z^{8}-(49+18 i) z^{7}-(99-23 i) z^{6}-(88-115 i) z^{5}+$ $(46+154 i) z^{4}+(168+18 i) z^{3}+(96-140 i) z^{2}-(32+104 i) z-(32+16 i)$.

While the number of permutation matrices for $n=6,7,8,9$ are $720,5040,40320$, 362880 , the corresponding number of alternating sign matrices are 7436, 218348, 10850216, 911835460. In the following sections we extend polynomial assignment to more generality, thereby getting an even larger number of matrices.

\section{Doubly Stochastic Matrices and Polynomiography}

Our motivation here is to assign polynomials and polynomiography to an even larger class of matrices than the class of permutation matrices and eventually in subsequent sections, even to the larger class than the class of alternating sign matrices. We begin with a definition.

Definition 2 For each $k=1, \ldots, n$ let $\Omega_{k}$ denote the set of all $n \times n$ matrices whose entries are from the set $\{0,1, \ldots, k\}$ and such that the sum of the entries of each row and column is $k$.

We wish to associate polynomials to matrices in $\Omega_{k}$. Since an element of $\Omega_{k}$ is the sum of $k$ permutation matrices, each having a corresponding permutation polynomial, it is natural to associate to such an element the polynomial that is the product of these permutation polynomials. For instance, a matrix in $\Omega_{2}$ corresponds to the sum of two permutation polynomials. There are 18 such matrices, six of which come directly from scalar multiples of the corresponding size permutation matrices. These are

$$
\begin{aligned}
& \left(\begin{array}{lll}
2 & 0 & 0 \\
0 & 2 & 0 \\
0 & 0 & 2
\end{array}\right),\left(\begin{array}{lll}
2 & 0 & 0 \\
0 & 0 & 2 \\
0 & 2 & 0
\end{array}\right),\left(\begin{array}{lll}
0 & 2 & 0 \\
2 & 0 & 0 \\
0 & 0 & 2
\end{array}\right),\left(\begin{array}{lll}
0 & 0 & 2 \\
2 & 0 & 0 \\
0 & 2 & 0
\end{array}\right),\left(\begin{array}{lll}
0 & 2 & 0 \\
0 & 0 & 2 \\
2 & 0 & 0
\end{array}\right),\left(\begin{array}{lll}
0 & 0 & 2 \\
0 & 2 & 0 \\
2 & 0 & 0
\end{array}\right) \\
& \left(\begin{array}{lll}
0 & 1 & 1 \\
1 & 0 & 1 \\
1 & 1 & 0
\end{array}\right),\left(\begin{array}{lll}
0 & 1 & 1 \\
1 & 1 & 0 \\
1 & 0 & 1
\end{array}\right),\left(\begin{array}{lll}
1 & 0 & 1 \\
0 & 1 & 1 \\
1 & 1 & 0
\end{array}\right),\left(\begin{array}{lll}
1 & 1 & 0 \\
0 & 1 & 1 \\
1 & 0 & 1
\end{array}\right),\left(\begin{array}{lll}
1 & 0 & 1 \\
1 & 1 & 0 \\
0 & 1 & 1
\end{array}\right),\left(\begin{array}{lll}
1 & 1 & 0 \\
1 & 0 & 1 \\
0 & 1 & 1
\end{array}\right) \\
& \left(\begin{array}{lll}
2 & 0 & 0 \\
0 & 1 & 1 \\
0 & 1 & 1
\end{array}\right),\left(\begin{array}{lll}
0 & 2 & 0 \\
1 & 0 & 1 \\
1 & 0 & 1
\end{array}\right),\left(\begin{array}{lll}
0 & 0 & 2 \\
1 & 1 & 0 \\
1 & 1 & 0
\end{array}\right),\left(\begin{array}{lll}
0 & 1 & 1 \\
2 & 0 & 0 \\
0 & 1 & 1
\end{array}\right),\left(\begin{array}{lll}
1 & 0 & 1 \\
0 & 2 & 0 \\
1 & 0 & 1
\end{array}\right),\left(\begin{array}{lll}
1 & 1 & 0 \\
0 & 0 & 2 \\
1 & 1 & 0
\end{array}\right) \\
& \left(\begin{array}{lll}
0 & 1 & 1 \\
0 & 1 & 1 \\
2 & 0 & 0
\end{array}\right),\left(\begin{array}{lll}
1 & 0 & 1 \\
1 & 0 & 1 \\
0 & 2 & 0
\end{array}\right),\left(\begin{array}{lll}
1 & 1 & 0 \\
1 & 1 & 0 \\
0 & 0 & 2
\end{array}\right)
\end{aligned}
$$



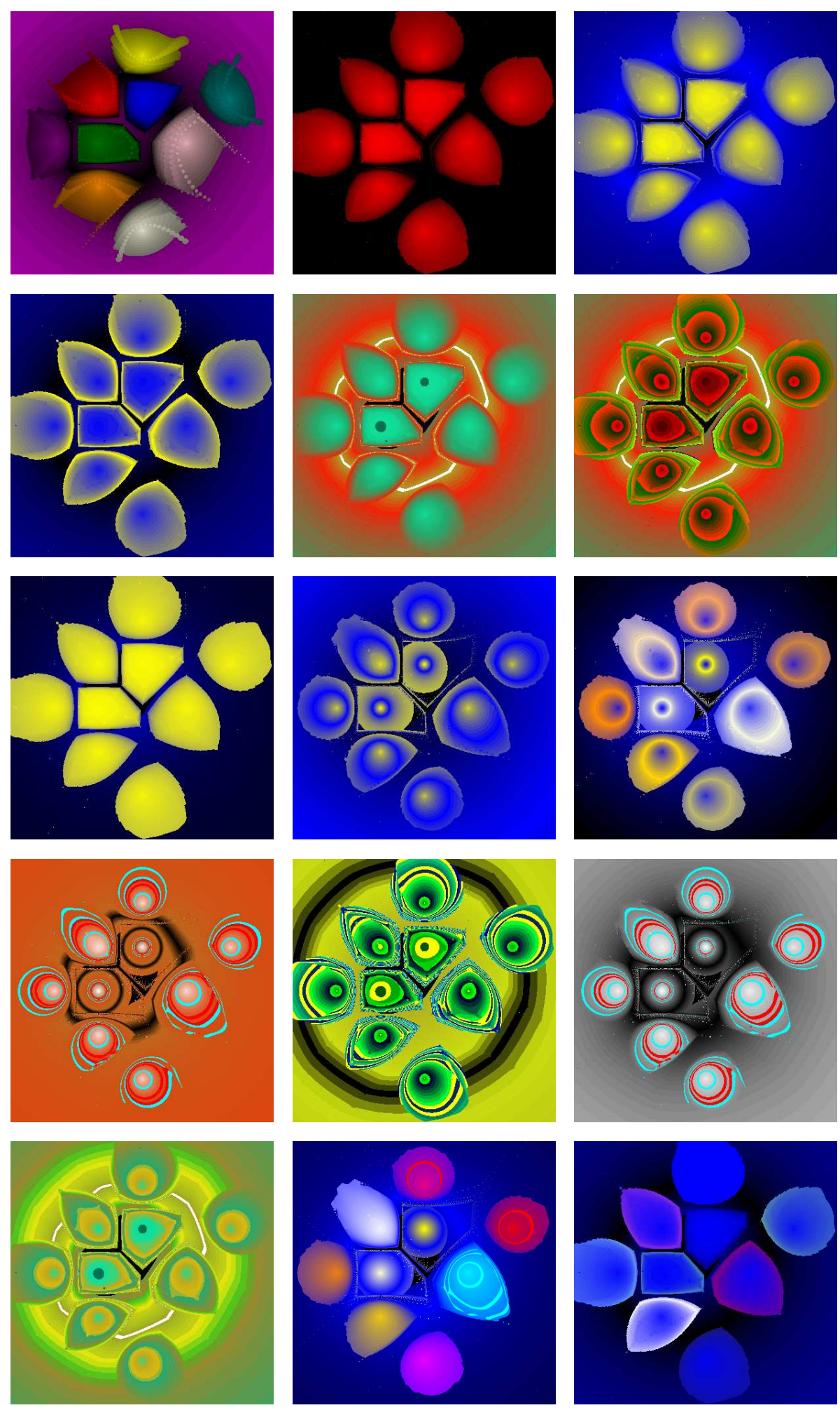

Figure 3: Polynomiographs corresponding to ASM on the cover of Bressoud's book [3]. 


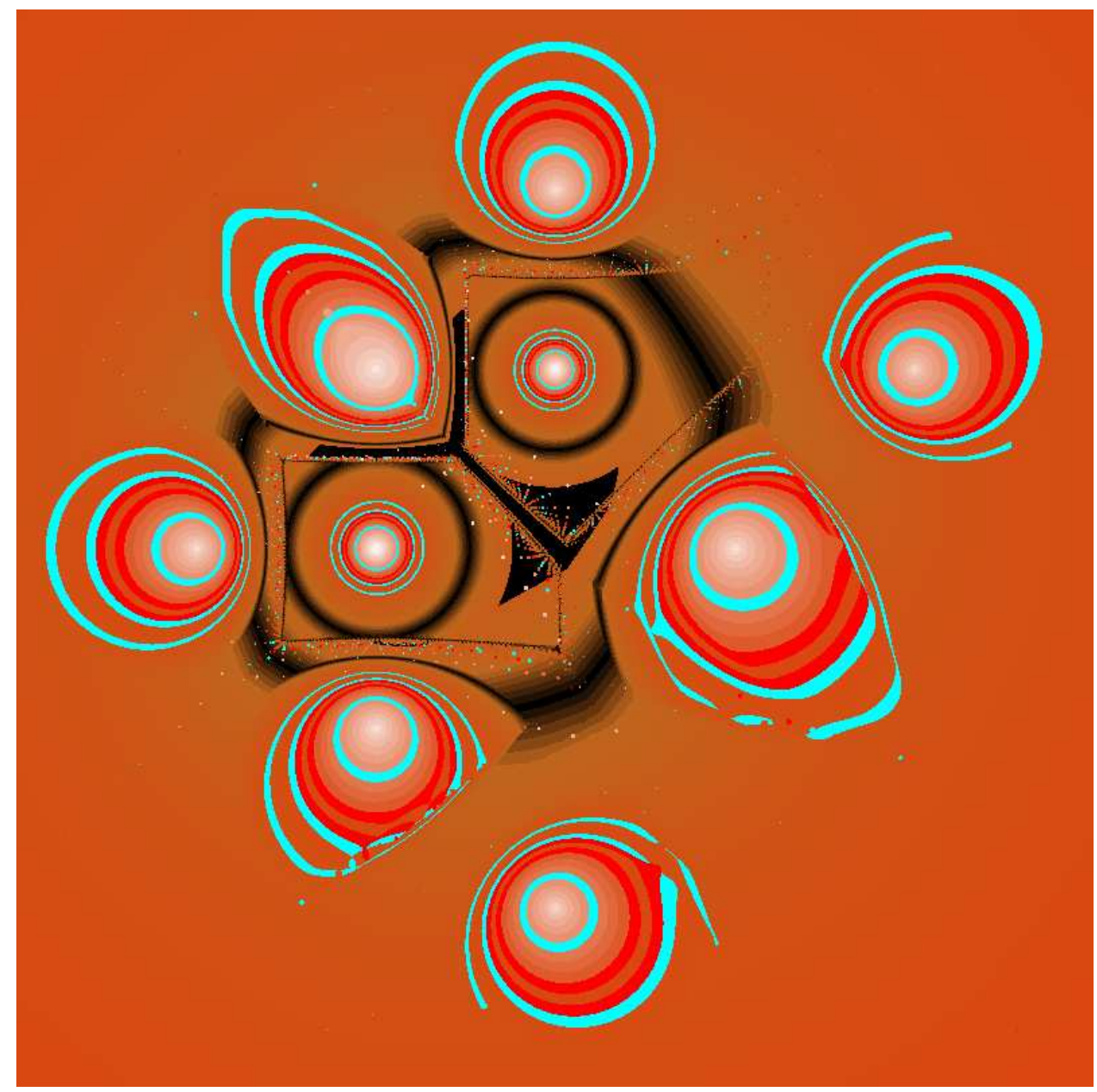

Figure 4: An enlarged polynomiograph. 
An $n \times n$ matrix $A=\left(a_{i j}\right)$ is doubly stochastic (DS) if all entries are nonnegative reals and the sum of the entries in each row equals the sum of the entries in each column and the common sum is one. These are significant matrices with numerous applications as the entries can be related to probabilities. We will now associate a corresponding polynomial to each such matrix. Specifically, given a doubly stochastic matrix (DSM) $A=\left(a_{i j}\right)$, the corresponding doubly stochastic polynomial is defined as follows. Let $\bar{A}$ and $\theta_{i j}$ be as defined previously. Then set

$$
P_{A}(z)=\prod_{\bar{a}_{i j}>0}\left(z-\bar{a}_{i j} \theta_{i j}\right)
$$

As an example, for $n=2$ the following are two special cases derived as linear combinations of the 2 by 2 permutation matrices with corresponding polynomiographs.

$$
\begin{gathered}
\frac{1}{2}\left(\begin{array}{ll}
1 & 0 \\
0 & 1
\end{array}\right)+\frac{1}{2}\left(\begin{array}{ll}
0 & 1 \\
1 & 0
\end{array}\right), \quad\left(z-\frac{(1+i)}{2}\right)\left(z-\frac{(1+2 i)}{2}\right)\left(z-\frac{(2+i)}{2}\right)\left(z-\frac{(2+2 i)}{2}\right) \\
\frac{1}{3}\left(\begin{array}{ll}
1 & 0 \\
0 & 1
\end{array}\right)+\frac{2}{3}\left(\begin{array}{ll}
0 & 1 \\
1 & 0
\end{array}\right), \quad\left(z-\frac{2(1+i)}{3}\right)\left(z-\frac{(1+2 i)}{3}\right)\left(z-\frac{(2+i)}{3}\right)\left(z-\frac{2(2+2 i)}{3}\right) .
\end{gathered}
$$
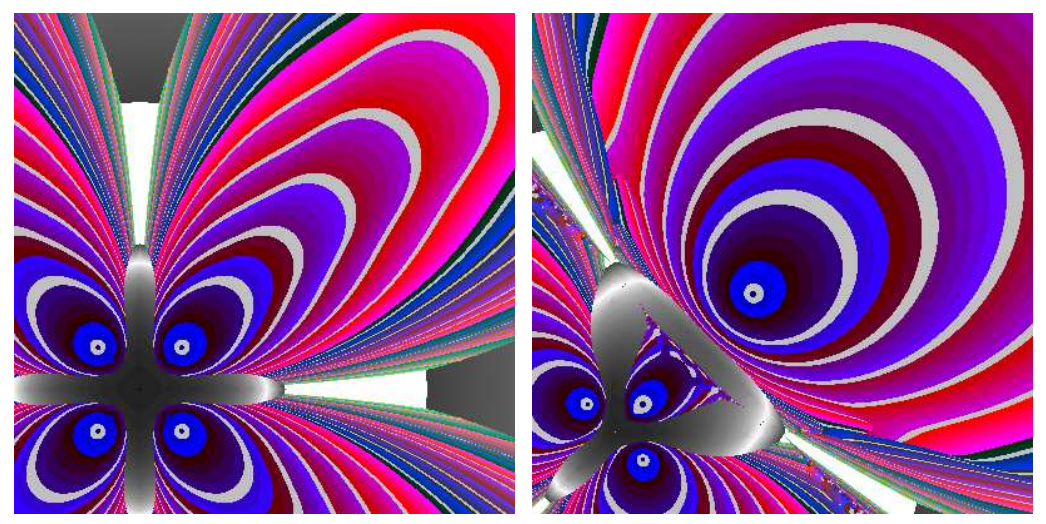

Figure 5: Polynomiographs corresponding to two convex combinations.

The left-hand-side polynomiograph in Figure 4 when drawn in appropriate window, would be similar to the polynomiograph of the polynomial having roots at $1+i, 1+2 i$, $2+i, 2+2 i$, in other words, the product of the complex permutation polynomials of order two. More precisely, the figure would be a homothetic translation of a corresponding polynomiograph for this product polynomial. This will be proved formally. First, this is used to justify the definition of doubly stochastic polynomials and their polynomiography; second, in conjunction with the Birkhoff-von Neumann theorem, it suggests that polynomiography of permutation matrices form building blocks for approximate polynomiography of doubly stochastic matrices. Permutation matrices and, more generally, doubly stochastic matrices give rise to a rich source of symmetry and art-making via polynomiography. In what follows we first prove an important property in the use of the Basic Family. 


\section{A Symmetry-Preserving Property}

Consider a complex polynomial in its product form

$$
p(z)=\left(z-z_{1}\right)\left(z-z_{2}\right) \cdots\left(z-z_{n}\right) .
$$

For a given real or complex scalar $\alpha$, let

$$
p_{\alpha}(z)=\left(z-\alpha z_{1}\right)\left(z-\alpha z_{2}\right) \cdots\left(z-\alpha z_{n}\right) .
$$

Consider Newton's iterate at $z_{0}$ with respect to $p(z)$. This is the same as $\alpha$ times Newton's iterate with respect to $p_{\alpha}(z)$ at $\alpha z_{0}$. More precisely, if

$$
N_{\alpha}(z)=z-\frac{p_{\alpha}(z)}{p_{\alpha}^{\prime}(z)}, \quad N(z)=N_{1}(z),
$$

then

$$
N_{\alpha}(\alpha z)=\alpha z-\frac{p_{\alpha}(\alpha z)}{p_{\alpha}^{\prime}(\alpha z)}=\alpha N(z) .
$$

More generally, this property can be stated for each member of the Basic Family.

Theorem 3 Let $\alpha$ be a real or complex scalar. Let $D_{m, \alpha}(z)$ denote $D_{m}(z)$ as applied to $p_{\alpha}(z)$, and

$$
B_{m, \alpha}(z)=z-p_{\alpha}(z) \frac{D_{m-2, \alpha}(z)}{D_{m-1, \alpha}(z)},
$$

then for any $z$ we have

$$
B_{m, \alpha}(\alpha z)=\alpha B_{m}(z)
$$

Proof. For each $k=0, \ldots, n$ we have

$$
p_{\alpha}^{k}(\alpha z)=\alpha^{n-k} p^{k}(z) .
$$

Using the recurrence relation (3) it is easy to prove the identity

$$
D_{m, \alpha}(\alpha z)=\alpha^{m(n-1)} D_{m}(z) .
$$

Thus

$$
\frac{D_{m-2, \alpha}(\alpha z)}{D_{m-1, \alpha}(\alpha z)}=\alpha^{-n+1} \frac{D_{m-2}(z)}{D_{m-1}(z)} .
$$

Since $p_{\alpha}(\alpha z)=\alpha^{n} p(z)$ the proof now follows. Q.E.D.

The implication of the theorem is the following.

Theorem 4 Under a fixed polynomiography technique two polynomiographs of $p(z)$ and $P_{\alpha}(z)$, except for a rotation, are homothetically equivalent.

Writing $\alpha$ in polar form as $r e^{\mathrm{i} \theta}$, the angle of rotation is $\theta$ and the homothetic ratio is $r$.

THE ELECTRONIC JOURNAL OF COMBINATORICS 18(2) (2011), \#P24 


\section{Polynomiography for Latin Squares and Sudoku}

An $n \times n$ Latin Square $L$ can be described as a matrix having entries from the set $\{1,2, \ldots, n\}$, where in each row and each column there is precisely one occurrence of each entry $i$. Since the sum of each row and each column is $s=n(n+1) / 2$, dividing by this number results in a doubly stochastic matrix. Thus, by applying Theorem 4, we conclude that the polynomiography of a Latin Square $L$ is homothetically equivalent to the polynomiography of the doubly stochastic matrix $\alpha L$, where $\alpha=1 / s$. There are a huge number of Latin Squares even for small values of $n$. There are known lower bounds on the number of $n \times n$ Latin Squares. Below we describe a way to estimate the number of $n \times n$ Latin Squares.

Let $E$ denote the $n \times n$ matrix of ones. This matrix can be decomposed into the sum of $n$ disjoint permutation matrices $\Pi_{1}, \ldots \Pi_{n}$, where by disjoint we mean $\Pi_{i}$ and $\Pi_{j}$ do not have a one in the same matrix entry. This decomposition can be achieved via a greedy algorithm.

Pick any permutation $\Pi_{1}$ and consider $E_{1}=E-\Pi_{1}$. The sum of the entries in each row of $E_{1}$ is $n-1$. Thus, $E /(n-1)$ is doubly stochastic and by the Birkhoff-von Neumann theorem it can be written as a convex combination of permutation matrices. None of these permutation matrices can have a positive entry where $\Pi_{1}$ is one. This is clear, since otherwise we get a positive entry in $E_{1}$ where there is a zero, a contradiction. This means we can pick another permutation matrix $\Pi_{2}$ disjoint from $\Pi_{1}$ and repeat this approach. Rather than invoking the Birkhoff-von Neumann theorem we can select $\Pi_{2}$ at random: select a random positive entry in $E_{1}$ and delete the corresponding row and columns. Then repeat this for the $(n-1) \times(n-1)$ matrix that remains. In this fashion, we can select a set of $n$ locations in $E_{1}$ that correspond to a permutation matrix $\Pi_{2}$ disjoint from $\Pi_{1}$. From the repeated application of this approach, we obtain a decomposition of $E$ as the sum of $n$ disjoint permutations. We can then label them as $1, \ldots n$. This labeling can be achieved in $n$ ! ways. Therefore we have the following proposition.

Proposition 1 The number of Latin Squares is equal to $n$ ! times the number of ways $E$ can be written as the sum of $n$ disjoint permutation matrices and where no two such representations have an identical set of permutation matrices.

The number of permutation matrices and alternating sign matrices for $n=6 ; 7 ; 8 ; 9$ were given earlier. In contrast, the number of Latin Squares is much larger. Even for $n=6$ their number is already large, 812851200, and for $n=7 ; 8 ; 9$ their number is respectively (see [13, 19] or Wikipedia on Latin Squares), $61479419904000 \approx 6.1 \times 10^{13}$,

$$
108776032459082956800 \approx 1.08 \times 10^{20}, \quad 5524751496156892842531225600 \approx 5.5 \times 10^{28} .
$$

As another source for polynomiography of special classes of doubly stochastic matrices, we can consider Sudoku solution matrices. The number of these $9 \times 9$ matrices is known to be (see Wikipedia on Mathematics of Sudoku)

$$
6670903752021072936960 \approx 6.67 \times 10^{21} .
$$


In summary, in this section we have shown how we may associate polynomiography to a large class of doubly stochastic matrices. Our next goal is to associate approximate polynomiography to doubly stochastic matrices where this polynomiography can be achieved efficiently from already computed polynomiographs of permutation matrices or alternating sign matrices.

\section{Approximate Polynomiography for a DSM}

By the Birkhoff-von Neumann theorem, [2] and [16], every doubly stochastic matrix is the convex combination of permutation matrices. Suppose we are given a doubly stochastic matrix $A$ as a convex combination of permutation matrices

$$
A=\sum_{i=1}^{k} \alpha_{i} \Pi_{i}, \quad \sum_{i=1}^{k} \alpha_{i}=1, \quad \alpha_{i} \geq 0,
$$

where for each $i=1, \ldots, k, \Pi_{i}$ is a permutation matrix. Suppose that we have computed distinct polynomiographs $P_{i}, i=1, \ldots, k$, corresponding to the permutation polynomial $p_{\Pi_{i}}$ and where they all represent a rectangle with the same coordinates. We wish to use these polynomiographs to efficiently compute an approximate polynomiograph for $A$ itself. We may assume that a common method in polynomiography is used to generate the polynomiographs.

We can think of $P_{i}$ as matrices of pixels with colors, where the color is a certain number assigned based on the roots of $p_{\Pi_{i}}$, and the particular polynomiography method used. Suppose the roots corresponding to $p_{\Pi_{i}}$ are $r_{i 1}, \ldots, r_{i s_{i}}$, with assigned colors $c_{i 1}, \ldots, c_{i s_{i}}$. Suppose that for $i=1, \ldots, k$ the color associated with a pixel with respect to $P_{i}$ is $c_{i}$, and it is based on a root $r_{i j_{i}}$ of $p_{\Pi_{i}}$. Suppose that the distance of the pixel to this root is $d_{i}$. We wish to associate a color to this pixel based on a certain convex hull property. One possible way is according to the function that takes into account the color that is a linear combination of these, say a weighted color based on the proximity of the pixel to $r_{i j_{i}}$ :

$$
\left(\sum_{i=1}^{k} \alpha_{i} \frac{c_{i}}{d_{i}}\right)\left(\sum_{i=1}^{k} \frac{1}{d_{i}}\right)^{-1} .
$$

The formula takes into account the shifting of roots of $p_{\Pi_{i}}$ when $\Pi_{i}$ is multiplied by $\alpha_{i}$ and it implies that when the pixel is closest to a root $r_{i j_{i}}, \alpha_{i} c_{i}$ will be more prominent than other colors. There are cases where a pixel may not belong to any basin of attraction for one or more $P_{i}$, however using the convergence properties of the Basic Family it can be argued that under certain modes of polynomiography, for instance using individual $B_{m}(z)$ when $m$ is large, the above formula would be reasonably good. There are other ways to define approximate polynomiographs, however we postpone detailed discussion until later. 


\subsection{Decomposition Algorithm for a DSM}

Suppose $A$ is a doubly stochastic matrix of order $n$. We wish to write it as a convex combination of permutation matrices, a valid representation due to the Birkhoff von Neumann theorem. In what follows, we describe an algorithm for computing such a decomposition.

We wish to decompose $A$ as the sum of permutations matrices. We offer a procedure that is essentially the von Neumann proof of the Birkhoff-von Neumann theorem [16]. Let the smallest entry of $A$ be denoted by $\delta_{1}$, corresponding to an entry $a_{i_{1} j_{1}}$. Next find a permutation that contains the location $\left(i_{1}, j_{1}\right)$. This can be done by considering the bipartite graph $G=(U, V, E)$, where $U$ is the set of rows of $A$ and $V$ the set of columns, and $E$ consists of the edges $\left(u_{i}, v_{j}\right)$ where $a_{i j}>1$. Next, we find a perfect matching on the edges of $E$ that contains the edge $\left(u_{i_{1}}, v_{j_{1}}\right)$. A perfect matching is a set of edges incident to all the vertices in $G$ and where no two distinct edges have a common vertex. This perfect matching corresponds to a permutation matrix $\Pi_{1}$. Let $A_{1}=A-\delta_{1} \Pi_{1}$. The row and column sums in $A_{1}$ are all $1-\delta_{1}$. If this number is zero, then $A$ is already a permutation matrix. If not, set $A_{2}=\frac{1}{1-\delta_{1}} A_{1}$. Then $A_{2}$ is doubly stochastic. Let $\delta_{2}$ be the smallest entry of $A_{2}$, and analogously, let $\Pi_{2}$ a permutation matrix that contains the location containing $\delta_{2}$. Let $A_{3}=A_{2}-\delta_{2} \Pi_{2}$, and if $1-\delta_{2}$ is not zero, set $A_{4}=\frac{1}{1-\delta_{2}} A_{3}$. Repeating this process, we are able to write $A$ as

$$
A=\delta_{1} \Pi_{1}+\left(1-\delta_{1}\right) \delta_{2} \Pi_{2}+\cdots+\prod_{i=1}^{k-2}\left(1-\delta_{i}\right) \delta_{k-1} \Pi_{k-1}+\prod_{i=1}^{k}\left(1-\delta_{k}\right) A_{k},
$$

where $\Pi_{i}, i=1, \ldots, k-1$ is a permutation matrix, and $\left(1-\delta_{i}\right)>0$, for $i=1, \ldots, k$, also $A_{k}$ itself is a permutation matrix, say $\Pi_{k}$. Summing over all entries in $A$ gives $n$. Also, summing over the right-hand side matrices, we must get $n$. From this we conclude

$$
\delta_{1}+\left(1-\delta_{1}\right) \delta_{2}+\cdots+\prod_{i=1}^{k-2}\left(1-\delta_{i}\right) \delta_{k-1}+\prod_{i=1}^{k}\left(1-\delta_{k}\right)=1 .
$$

This procedure gives an algorithmic decomposition of $A$ as a convex combination of permutation matrices. In fact, the number of permutations in the decomposition is bounded above by the number of positive entries in $A$.

\section{Polynomiography for Matrices in the ASM Poly- tope}

The ASM polytope of order $n$ is the convex hull of $n \times n$ alternating sign matrices. It is denoted by $A S M_{n}$ and has been recently characterized by Striker [20]. Clearly this polytope contains the convex hull of the $n \times n$ doubly stochastic polytope, the Birkhoff polytope. Here we associate to an element $A=\left(a_{i j}\right)$ in $A S M_{n}$ a polynomial as follows. With $\bar{A}$ and $\theta_{i j}$ as defined previously, see (6) and (7), let 


$$
P_{A}(z)=\prod_{\bar{a}_{i j}>0}\left(z-\bar{a}_{i j} \theta_{i j}\right) \times \prod_{\bar{a}_{i j}<0}\left(z-\bar{a}_{i j} \theta_{i j}\right)^{2} .
$$

Striker [20] has shown that the vertices of the $A S M_{n}$ are precisely $n \times n$ alternating sign matrices. As in the case of doubly stochastic matrices it would be desirable to have an algorithm that would decompose a given member of the ASM polytope into a convex combination of alternating sign matrices. Given such decomposition, one can apply the approximate polynomiography approach as described for doubly stochastic matrices. We will describe such a decomposition algorithm. In fact, the algorithm is a direct generalization of the one for doubly stochastic matrices described before. First, we describe Striker's description of $A S M_{n}$.

Theorem 5 (Striker[20]) The convex hull of $n \times n$ alternating sign matrices consists of all $n \times n$ real matrices $X=\left(x_{i j}\right)$ such that:

$$
\begin{gathered}
0 \leq \sum_{i=1}^{i^{\prime}} x_{i j} \leq 1, \quad \forall 1 \leq i^{\prime} \leq n, \quad 1 \leq j \leq n . \\
0 \leq \sum_{j=1}^{j^{\prime}} x_{i j} \leq 1, \quad \forall 1 \leq j^{\prime} \leq n, \quad 1 \leq i \leq n . \\
\sum_{i=1}^{n} x_{i j}=1, \quad \forall 1 \leq j \leq n . \\
\sum_{j=1}^{n} x_{i j}=1, \quad \forall 1 \leq i \leq n .
\end{gathered}
$$

To describe the decomposition algorithm, we first state a result that is a generalization of a known result for the doubly stochastic polytope and follows from the fact that the optimal solution of a linear program is attained at a vertex.

Proposition 2 Let $A=\left(a_{i j}\right)$ lie in $A S M_{n}$. Suppose for a given pair of indices $i_{0}, j_{0}$, $a_{i_{0} j_{0}}>0$. Then there exists an $n \times n$ alternating sign matrix $B=\left(b_{i j}\right)$ such that $b_{i j} \neq 0$ implies $a_{i j} \neq 0$. Furthermore, such $B$ can be computed as an optimal solution of the linear program

$$
\max \left\{x_{i_{0} j_{0}}: X=\left(x_{i j}\right) \in A S M_{n}, \quad x_{i j}=0, \quad \text { if } \quad a_{i j}=0\right\} .
$$

We next state a property of $A S M_{n}$.

Theorem 6 Suppose $A=\left(a_{i j}\right)$ lies in $A S M_{n}$. Let

$$
\delta=a_{i_{0}, j_{0}}=\min \left\{a_{i j}: 1 \leq i, j \leq n, a_{i j}>0\right\} .
$$

Let $B=\left(b_{i j}\right)$ be an ASM where $b_{i_{0}, j_{0}}=1$ and such that if $b_{i j} \neq 0$ then $a_{i j} \neq 0$. Then

$$
X=\frac{1}{1-\delta}(A-\delta B) \in A S M_{n}
$$


Proof. We need to show that $X=\left(x_{i j}\right)$ satisfies all the four types of constraints in Theorem 5. Clearly, $X$ satisfies the last two types of constraints in the theorem. We show it satisfies the first types of constraints. The second types follow from a symmetry argument. Consider a particular pair of indices $i^{\prime}$ and $j$. Since $A$ and $B$ are in $A S M_{n}$, Theorem 5 implies

$$
0 \leq \sum_{i=1}^{i^{\prime}} a_{i j} \leq 1, \quad 0 \leq \sum_{i=1}^{i^{\prime}} b_{i j} \leq 1
$$

But since $b_{i j}=0, \pm 1$ it must be the case that either $\sum_{i=1}^{i^{\prime}} b_{i j}=0$, or $\sum_{i=1}^{i^{\prime}} b_{i j}=1$. Thus

$$
\sum_{i=1}^{i^{\prime}} x_{i j}=\sum_{i=1}^{i^{\prime}} a_{i j}-\delta \sum_{i=1}^{i^{\prime}} b_{i j} \leq 1
$$

Next, we show $\sum_{i=1}^{i^{\prime}} x_{i j} \geq 0$. As argued above $\sum_{i=1}^{i^{\prime}} b_{i j}$ is either 0 or 1 . If it is zero, then since $\sum_{i=1}^{i^{\prime}} a_{i j} \geq 0$, we have $\sum_{i=1}^{i^{\prime}} x_{i j} \geq 0$. If $\sum_{i=1}^{i^{\prime}} b_{i j}=1$, then there must exist $i \leq i^{\prime}$ such that $a_{i j}>0$. But then by definition of $\delta$ we must have $a_{i j} \geq \delta$. This proves $\sum_{i=1}^{i^{\prime}} x_{i j} \geq 0$. The proof is complete. Q.E.D.

Corollary 1 Suppose $A=\left(a_{i j}\right)$ lies in $A S M_{n}$, and has $m$ positive entries. Then for some index $k \leq m$ we can compute ASMs $X_{i}, i=1, \ldots, k$, and constants $0<\delta_{i}<1$, $i=1, \ldots, k$, such that

$$
A=\delta_{1} X_{1}+\left(1-\delta_{1}\right) \delta_{2} X_{2}+\cdots+\prod_{i=1}^{k-2}\left(1-\delta_{i}\right) \delta_{k-1} X_{k-1}+\prod_{i=1}^{k}\left(1-\delta_{k}\right) X_{k},
$$

where

$$
\delta_{1}+\left(1-\delta_{1}\right) \delta_{2}+\cdots+\prod_{i=1}^{k-2}\left(1-\delta_{i}\right) \delta_{k-1}+\prod_{i=1}^{k}\left(1-\delta_{k}\right)=1 .
$$

In particular, $A$ is a convex combination of $X_{1}, \ldots, X_{k}$.

Proof. The justification is analogous to the case of a doubly stochastic matrix and the corresponding algorithm described earlier. Let $\delta_{1}$ be the smallest positive entry of $A=\left(a_{i j}\right)$ corresponding to an entry $a_{i_{1} j_{1}}$. Let $\Pi_{1}$ be an alternating sign matrix that is an optimal solution to the linear program

$$
\max \left\{x_{i_{1} j_{1}}: X=\left(x_{i j}\right) \in A S M_{n}, \quad x_{i j}=0, \quad \text { if } \quad a_{i j}=0\right\} .
$$

Let $A_{1}=A-\delta_{1} \Pi_{1}$. The row and column sums in $A_{1}$ are all $1-\delta_{1}$. If this number is zero, then $A$ is already an alternating sign matrix. If not, set $A_{2}=\frac{1}{1-\delta_{1}} A_{1}$. Then applying Theorem $6, A_{2}=\left(a_{i j}^{(2)}\right)$ lies in $A S M_{n}$. Let $\delta_{2}$ be the smallest positive entry of $A_{2}$ corresponding to an entry $a_{i_{2} j_{2}}^{(2)}$. Let $\Pi_{2}$ be an alternating sign matrix that is an optimal solution to the linear program

$$
\max \left\{x_{i_{2} j_{2}}: X=\left(x_{i j}\right) \in A S M_{n}, \quad x_{i j}=0, \quad \text { if } \quad a_{i j}^{(2)}=0\right\} .
$$


Let $A_{3}=A_{2}-\delta_{2} \Pi_{2}$, and if $1-\delta_{2}$ is not zero, set $A_{4}=\frac{1}{1-\delta_{2}} A_{3}$. Repeating this process, we are able to decompose $A$ as claimed with the number of alternating sign matrices in the decomposition is bounded above by the number of positive entries in A. Q.E.D.

Remark 1 Striker [20] gives a direct proof that each feasible point in the ASM polytope can be written as a convex combination using the terminology of circuits. Our approach for the decomposition relies on Theorem 5 , but then directly employs linear programming theory. Indeed, for small values of $n$ one can directly apply LP software. In view of Striker's description of the ASM polytope we may also conclude that the decomposition in the above corollary can be accomplished in fully polynomial time. Such decomposition is clearly not unique. It would be interesting to give analysis of the complexity for an efficient algorithm and its dependence on $n$ and $m$.

As an example, suppose we wish to associate polynomiography to the $3 \times 3$ Latin Square below, where we have scaled it by 6 to get a doubly stochastic matrix.

$$
A=\frac{1}{6}\left(\begin{array}{lll}
2 & 1 & 3 \\
1 & 3 & 2 \\
3 & 2 & 1
\end{array}\right)
$$

We apply the decomposition algorithm. It is easy to check that the algorithm leads to the following as one possible decomposition.

$$
A=\frac{1}{6}\left(\begin{array}{ccc}
0 & 1 & 0 \\
1 & -1 & 1 \\
0 & 1 & 0
\end{array}\right)+\frac{5}{6} \frac{1}{5}\left(\begin{array}{ccc}
1 & 0 & 0 \\
0 & 0 & 1 \\
0 & 1 & 0
\end{array}\right)+\frac{5}{6} \frac{4}{5} \frac{1}{4}\left(\begin{array}{lll}
1 & 0 & 0 \\
0 & 1 & 0 \\
0 & 0 & 1
\end{array}\right)+\frac{5}{6} \frac{4}{5} \frac{3}{4}\left(\begin{array}{ccc}
0 & 0 & 1 \\
0 & 1 & 0 \\
1 & 0 & 0
\end{array}\right) .
$$

In the above example we have purposely used an ASM in the decomposition but clearly we could have obtained a decomposition purely in terms of permutation matrices. As the example implies, in terms of approximate polynomiography of a doubly stochastic matrix, an ASM matrix can come to play a role if so desired.

\subsection{Diagonal Scaling into an ASM}

Finally, we consider polynomiography to extensions of more general matrices. First we give some definitions.

Definition 3 Two $n \times n$ matrices $A=\left(a_{i j}\right)$ and $B=\left(b_{i j}\right)$ have the same pattern if $a_{i j}$ and $b_{i j}$ are either both zero, both positive, or both negative.

Definition 4 An $n \times n$ matrix $A=\left(a_{i j}\right)$ has the $A S M_{n}$ sign property if there exists $B \in A S M_{n}$ such that $A$ has the same pattern as $B$.

Definition 5 An $n \times n$ matrix $A=\left(a_{i j}\right)$ having the $A S M_{n}$ sign property is diagonally scalable if there exist two $n \times n$ diagonal matrices $U$ and $V$ with positive diagonal entries, and a matrix $B \in A S M_{n}$ such that

$$
B=U A V
$$


Given $B=U A V$ we can associate a polynomial to $B$ as follows

$$
P_{B}(z)=\prod_{\bar{a}_{i j}>0}\left(z-u_{i} v_{j} \bar{a}_{i j} \theta_{i j}\right) \times \prod_{\bar{a}_{i j}<0}\left(z-u_{i} v_{j} \bar{a}_{i j} \theta_{i j}\right)^{2} .
$$

This allows association of polynomiography to a more general class of matrices than those in the ASM polytope. When $A$ has nonnegative entries, the corresponding diagonal scalability is a well-studied problem. In particular, the following facts are known about nonnegative matrices. First, a nonnegative matrix $A$ is diagonally scalable if and only if each nonnegative entry can be covered in a perfect matching. Second, the existence and computation of $U$ and $V$ can be achieved in polynomial time. Further, the so-called RAS algorithm gives rise to a practical algorithm. For these, see [17], [11], [12] and references therein. For a matrix $A$ having the $A S M_{n}$ sign property, the problem of deciding if it is diagonally scalable is most likely a challenging problem in itself. Two questions that can be posed are: (1) Is it true that a matrix $A$ has an $A S M_{n}$ sign pattern if and only if it can be diagonally scalable?, (2) If $A$ is diagonally scalable, then how efficiently can it be solved? Does the RAS method extend? Other research problems can be posed.

Concluding Remarks. In this article, we have associated complex polynomials to matrices in the Birkhoff and the ASM polytopes. Not only do these provide a rich source for polynomiography from the artistic point of view, but also a way to connect several distinct theoretical and algorithmic concepts to the alternating sign matrices. This makes possible the introduction of various concepts in interdisciplinary courses. Additionally, the investigation of ASM polynomials give rise to some seemingly interesting research problems. We have posed some in the context of matrix scaling. But clearly other research topics arise. For instance, the question of general convergence of complex permutation polynomials or complex alternating sign polynomials, say under Newton's method, is interesting from the point of view of dynamical systems. From the point of view of polynomiography itself, the implementation of the approximate polynomiography for an arbitrary member of the ASM polytope is the subject of our future work, especially in the anticipation that they will yield interesting images. We plan to exhibit online many polynomiography images relevant to this article that we have omitted due to space limitation, as well as new ones that would be possible as a result of implementing new algorithms.

Acknowledgements. I would like to thank an anonymous referee for very useful comments.

\section{References}

[1] A. F. Beardon, Iteration of Rational functions: Complex Analytic Dynamical Systems. Springer-Verlag, New York, 1991. 
[2] G. Birkhoff, Three observations on linear algebra, Univ. Nac. Tucumán Rev. Ser. A 5 (1946), pp. 147-151.

[3] D. Bressoud, Proofs and Confirmations: the story of the Alternating Sign Matrix Conjecture. Cambridge University Press, 1999.

[4] A. Cayley, The Newton-Fourier imaginary problem, American Journal of Mathematics, 2 (1879), p. 97.

[5] B. Kalantari, Generalization of Taylor's theorem and Newton's method via a new family of determinantal interpolation formulas and its applications, J. of Comp. and Appl. Math., 126 (2000), pp. 287-318.

[6] B. Kalantari, On homogeneous linear recurrence relations and approximation of zeros of complex polynomials, DIMACS Series in Discrete Mathematics and Theoretical Computer Science, Unusual Applications in Number Theory, Volume 64 (2004), pp. $125-143$.

[7] B. Kalantari, Polynomiography: From the Fundamental Theorem of Algebra to Art, Leonardo, Vol. 38, No. 3 (2005), pp. 233-238.

[8] B. Kalantari, Polynomial Root-Finding and Polynomiography, World Scientific, Hackensack, NJ, 2008.

[9] B. Kalantari, Permutation Matrices and Polynomiography, in Symmetry: Art and Science, Special Issues for Festival-Congress, (2010), pp. 130-133.

[10] B. Kalantari, Polynomial Root-Finding Methods Whose Basins of Attraction Approximate Voronoi Diagram, Discrete and Computational Geometry, (2011), available online.

[11] B. Kalantari and L. Khachiyan, On the complexity of nonnegative-matrix scaling, Linear Algebra and its Applications 240 (1996), pp. 87-103.

[12] B. Kalantari, I. Lari, F. Ricca, B. Simeone, On the complexity of general matrix scaling and entropy minimization via the RAS algorithm, Mathematical Programming, Series A, 112 (2007), pp. 371-401.

[13] B.D. McKay and I. M. Wanless, On the number of Latin squares, Ann. Comb. 9 (2005), 335-344.

[14] W. H. Mills, D. P. Robbins, and H. Rumsey, Jr., Alternating sign matrices and descending plane partitions, J. Combin. Theory Ser. A, 34 (1983), no. 3, pp. 340359.

[15] J. Milnor, Dynamics in One Complex Variable: Introductory Lectures, Vol 160, 3rd edn. Princeton University Press, New Jersey, 2006.

[16] J. von Neumann, A certain zero-sum two-person game equivalent to an optimal assignment problem, Ann. Math. Studies 28 (1953), pp. 5-12.

[17] U. G. Rothblum and H. Schneider, Scaling of matrices which have prescribed row sums and column sums via optimization, Linear Algebra and its Applications 114/115 (1989), pp. 737-764. 
[18] E. Schröder, On infinitely many algorithms for solving equations (German), Math. Ann., 2 (1870), pp. 317-365. (English translation by G.W. Stewart, TR-92-121, Institute for Advanced Computer Studies, University of Maryland, College Park, MD, 1992.)

[19] D. S. Stones, The many formulae for the number of Latin rectangles, Electronic Journal of Combinatorics, 17 (2010), A1, pp. 1-46.

[20] J. Striker, Alternating sign matrix polytope, Electronic Journal of Combinatorics, 16 (2009), R41, pp. 1-15.

[21] D. Zeilberger, Proof of the Alternating Sign Matrix conjecture, Electronic Journal of Combinatorics, 3, No. 2 (1996), R13, pp. 1-84. 